Proceedings of the 15th Czech and Slovak Conference on Magnetism, Košice, Slovakia, June 17-21 2013

\title{
Single Domain Wall Contribution to the Impedance of Amorphous Ferromagnetic Wire
}

\author{
J. Ziman*, V. ŠUHAJová, M. Kladivová \\ Department of Physics, Technical University of Košice, Letná 9, 04200 Košice, Slovakia
}

\begin{abstract}
An experiment, measuring the changes of impedance in a magnetic wire, caused by the presence of a single domain wall was proposed. The results obtained from a magnetic wire with small helical anisotropy show that for lower frequencies (up to about $1 \mathrm{MHz}$ ), the presence of wall causes an increase in impedance, very probably due to the wall displacement. As frequency increases, the influence of the wall presence on the magnetic state causes the impedance to decrease in adjacent domains. For frequencies close to $10 \mathrm{MHz}$ this effect overcomes the effect of wall displacement.
\end{abstract}

DOI: $10.12693 /$ APhysPolA.126.82

PACS: 75.30.Gw, 75.47.-m, 75.50.Kj, 75.60.-d, 84.37.+q

\section{Introduction}

Giant magneto-impedance (GMI) effect in thin ferromagnetic wires has been intensively studied for about two decades. This interest was strongly motivated by its promising application potential $[1,2]$. For better understanding of GMI effect many aspects have to be taken into account. One of them is the role of domain structure [3]. Our report presents an experiment in which we studied the contribution of a single boundary between circular domains to the impedance in $\mathrm{Co}_{68.2} \mathrm{Fe}_{4.3} \mathrm{Si}_{12.5} \mathrm{~B}_{15}$ amorphous ferromagnetic wire with diameter of $125 \mu \mathrm{m}$ (Unitika AC20).

Single domain wall experiments in this type of wire are presented in [4]. Our wire was annealed under simultaneous application of tensile and torsion stresses. In this way a small helical anisotropy was induced. The deviation of the easy axis from circular direction was very small, but this still enabled the creation of a single domain wall between circular domains (with negligible axial component of magnetization) in the wire [4]. This wall was created by applying an inhomogeneous axial field generated by Helmholtz coils connected in anti-parallel combination. The possibility of manipulating a single domain wall using an axial external field gives the opportunity to measure the wire impedance with and without presence of the wall, and thus to obtain its contribution to the wire impedance.

\section{Results and discussion}

The results of measurements presented in this paper were obtained on a sample $l_{2} \approx 2.2 \mathrm{~cm}$ long, treated as described above. The sample was positioned in an experimental set up which consisted of a long solenoid, which generated a homogeneous magnetic field $H$, and of a pair of Helmholtz coils connected in anti-parallel combination, which generated an inhomogeneous magnetic field $H_{H e}$. Two pairs of thin copper wires were attached to the wire

\footnotetext{
${ }^{*}$ corresponding author; e-mail: jan.ziman@tuke.sk
}

with silver paint. The first (inner) pair was attached approximately in its centre with the spacing of $l_{1} \approx 4 \mathrm{~mm}$ and the second (outer) one was attached to the wire ends.

For circular magnetization flux measurements, from which the wall position was derived (details can be found in [4]), both pairs of contacts were used.

These measurements were also used to find the position of the Helmholtz coils for which in zero field $H$ the wall is created approximately in the centre, between the inner pair of contacts. For impedance measurements the inner pair and a Lock-In amplifier were used.

The wall presence causes very small changes in the wire impedance. For this reason the following experimental procedure was used. Measurement of one point on the ascending branch of the impedance hysteresis loop starts at maximum-amplitude field $-H_{\max }$ generated by the solenoid, where impedance $Z_{\max }$ is measured. Then the field is changed to measuring value $H$ and impedance $Z_{1}$ is measured. In the next step the inhomogeneous field $H_{H e}$ is turned on for about 1 second. The magnitude of this field is high enough for the creation of a single domain wall in fields $H$ close to zero [4]. After the field $H_{H e}$ is turned off, the impedance $\mathrm{Z}_{2}$ is measured. The whole procedure is completed with a change of the field in the solenoid to the value $+H_{\max }$ and then back to the starting value $-H_{\max }$.

The wall position as a function of field $H$ is shown in Fig. 1. The position is plotted with respect to the centre of the wire and was derived from circular magnetic flux measurements between both the inner and the outer pairs of contacts, along the ascending branch of the hysteresis loop. Interpretation of these curves based on the model of single wall displacement is also given in this figure. Four regions can be identified. Field regions $\mathrm{A}$ and $\mathrm{D}$ where no wall is present, region $B$ in which the wall is present in the wire, but its position is outside the wire part between the inner contacts, and finally field region $\mathrm{C}$ where the wall is located between the inner contacts.

The change in impedance $\Delta Z_{1}=Z_{1}-Z_{\max }$ versus the applied axial field $H$ curves, measured at three different frequencies are shown in Fig. 2. In accordance with the type of the induced anisotropy, typical two-peak 


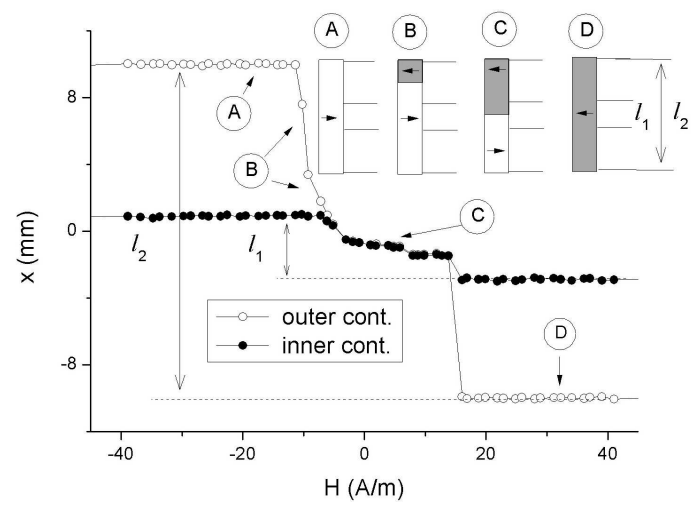

Fig. 1. Wall position as a function of axial field $H$ derived from the ascending branch of a circular magnetic flux loop [4], measured between both inner and outer pairs of contacts. Schematic interpretation of the wall localization for different regions of the applied field is given in the inset.

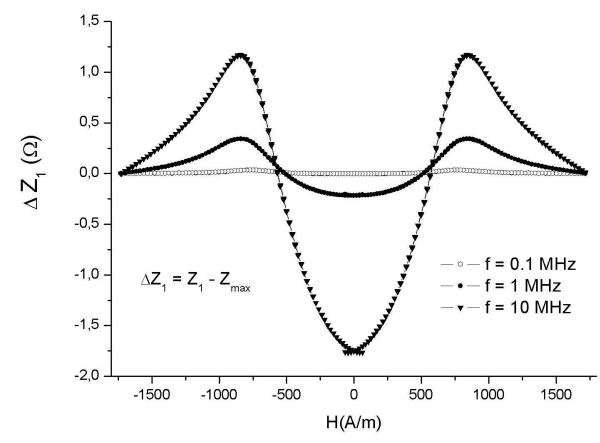

Fig. 2. Axial loops of impedance change $\Delta Z_{1}$ for three frequencies of ac current.

curves are observed [5]. These curves for $\Delta Z_{1}$ and also for $\Delta Z_{2}=Z_{2}-Z_{\max }$ for the low field region are shown in Fig. 3. For low frequency of $0.1 \mathrm{MHz}$ in Fig. 3a there is no visible change in $\Delta Z_{1}$, while an increase in $\Delta Z_{2}$ of about $5.8 \mathrm{~m} \Omega$ in the region $\mathrm{C}$ can be observed. Interesting behaviour can be seen in Fig. $3 \mathrm{~b}$ for the frequency of $3.5 \mathrm{MHz}$. For this frequency the magnitude of changes in $\Delta Z_{1}$ is comparable with those in $\Delta Z_{2}$. In the regions $\mathrm{A}$ and $\mathrm{D} \Delta Z_{2}$ follows the corresponding branches of the loop for $\Delta Z_{1}$. A decrease in impedance can be observed in the region $\mathrm{B}$ and an increase in the region $\mathrm{C}$. It can be seen that the presence of the wall can be detected from the impedance data, even when the wall is not located between the measuring points - the inner contacts. Very probably, the presence of the wall causes the magnetization in adjacent domains in the vicinity of the wall to rotate towards the circumferential direction, and due to decrease in permeability, the impedance also decreases. Since the induced helical anisotropy is dominant close to the wire surface, this effect becomes significant at higher frequencies. For the frequency of $10 \mathrm{MHz}$ (see Fig. 3c) the negative effect of magnetization changes due to wall presence overcomes the positive effect of domain wall displacement.

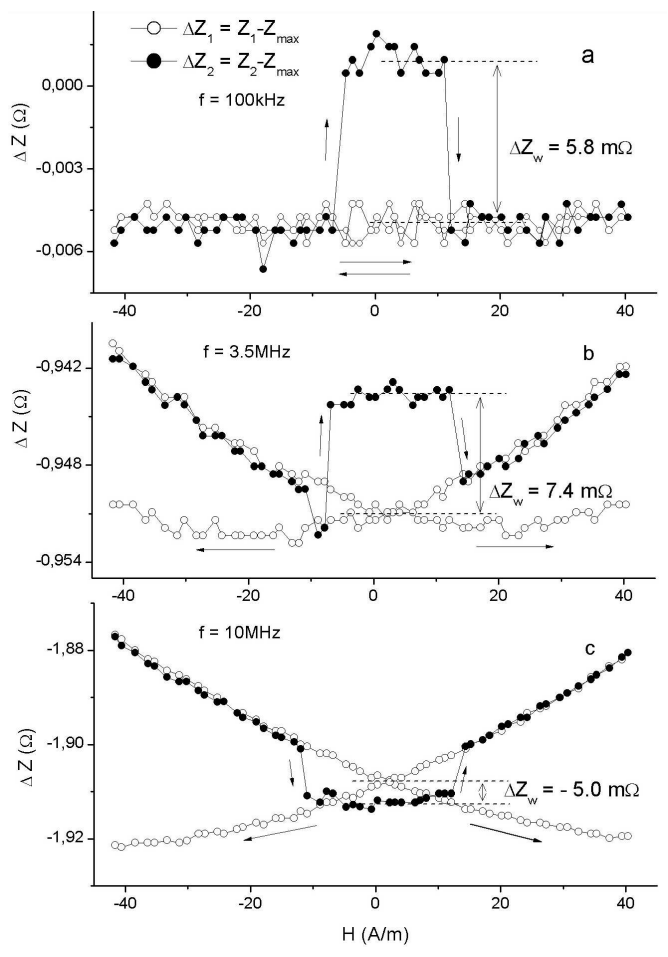

Fig. 3. Low field curves of impedance changes $\Delta Z_{1}$ and $\Delta Z_{2}$ for three frequencies of ac current. It is also indicated how the wall impedance $\Delta Z_{w}$ can be determined from these curves.

\section{Conclusions}

An experiment, making it possible to measure changes of impedance in a magnetic wire, caused by the presence of a single domain wall was proposed. The results obtained in this experiment show that for frequencies up to about $1 \mathrm{MHz}$ the wall impedance increases $\left(\Delta Z_{w} \approx 8 \mathrm{~m} \Omega\right)$ with increasing frequency probably due to the dominance of the positive wall displacement contribution. For higher frequencies the wall impedance decreases with frequency, and at frequencies close to $10 \mathrm{MHz}$ it even becomes negative. The most probable reason for this, is the decrease in impedance in adjacent domains separated by the domain wall.

\section{Acknowledgments}

This work was supported by the Slovak Research and Development Agency under contract No. APVV-0027-11 and also by VEGA grant No. 1/0778/12.

\section{References}

[1] M. Knobel, M. Vázquez, L. Kraus, Handbook of Magnetic Materials 15, 497 (2003).

[2] M.-H. Phan, H.-X. Peng, Prog. Mater. Sci. 53, 323 (2008).

[3] D.-X. Chen, J.L. Muñoz, A. Hernando, M. Vázquez, Phys. Rev. B 57, 10699 (1998).

[4] J. Ziman, J. Onufer, M. Kladivová, Physica B: Cond. Matt. 406, 3576 (2011).

[5] L.V. Panina, K. Mohri, K. Bushida, M. Noda, J. Appl. Phys. 76, 6198 (1994). 